\title{
ECG Feature Extraction Analysis System for Arrythmia Detection-A Review
}

\author{
Meghashree S J ${ }^{1}$, Shankar B B ${ }^{2}$, Praveen $\mathbf{J}^{3}$, Raghavendra Rao ${ }^{4}$ \\ M.Tech Student, VLSI Design and Embedded systems, AIET, Moodbidri, India ${ }^{1}$ \\ Associate professor, Electronics and communication, AIET, Moodbidri, India ${ }^{2}$ \\ Sr. Associate professor, Electronics and communication, AIET, Moodbidri, India ${ }^{3,4}$
}

\begin{abstract}
Electrocardiogram is an important tool in diagnosing the condition of the heart. Extracting the information from the Electrocardiograph is an important task in determining the variations of the electrical activity of the heart. ECG feature extraction plays a major significant role in diagnosing the most of the cardiac diseases. One among the major cardiac diseases is arrhythmia which is abrupt and abnormal heart beat. In case of arrhythmia heart doesn't pump sufficient blood required for the human body and sudden cardiac death may happen and this can even damage vital organs such as brain, heart, etc. of the body positions. So it is very much needed to determine conditions of arrhythmia and should take necessary measure before the patient reaches some serious condition. Hence in order to find out arrhythmia ECG signal should be analyzed. Analyzing the ECG signal manually is a tedious process so number of researches has been done recently for analyzing the ECG signal based on fuzzy logic method, artificial neural networks, genetic algorithms and support vector machines and using other analysis techniques. This proposed paper discusses different ECG analysis techniques and provides comparative study of various methods of such techniques proposed by researchers in the previous articles. as both an instruction set and as a template into which you can type your own text.
\end{abstract}

Keywords: Discrete wavelets transform, ECG signal, Feature extraction, QRS complex detection.

\section{INTRODUCTION}

An ECG plays a major role in the field of cardiology. ECG is an graphical representation of electrical activity of the atrial and ventricles, which are responsible for repolarization and depolarization. It contains important clinical information of heart. It is usually measured by placing an array of 12 different electrodes on the body surface of a patient; these electrodes are known as ECG leads. ECG signal has a time varying morphological characteristics named as $\mathrm{P}, \mathrm{T}$ waves and QRS complex. These waves will be of different amplitudes. Among these QRS complex wave has highest amplitude and specific shape and is the mid to high frequency wave. P and Q are the low frequency waves. According to this paper even small abnormality in these waves represents the diseases of the conduction system or diseases of the heart. Each of these waves has important relationship with the repolarization and depolarization of the heart. P-wave represents the depolarization of the atria, QRS complex represents the ventricular repolarization and T-wave is for ventricular repolarization. Extracting the information in the P-QRS-T waves is called feature extraction and it involves in determining the amplitude and intervals in the ECG signals. Variations in length and width of the QRS complex appear for short period of time and continue for indefinite periods of time. A lot of work has been done in the field of ECG signal analysis using various approaches and methods. The development of accurate and quick methods for automatic ECG feature extraction is of major importance. Therefore it is necessary that the feature extraction is to find as few as few properties as possible within the ECG signal that would allow successful abnormality detection and efficient prognosis. The basic principle of all methods involves transformation of ECG signal using different transformation techniques including Fourier transform, Hilbert transform, wavelet transform etc. physiological signals like ECG are considered to be quasi periodic in nature. They are of finite duration and non-stationary. Hence, a technique like Fourier transforms is inefficient for ECG. On the other and wavelet, is a very recent addition in this field of research, provides a powerful tool for extracting information from such signals. There has been use of both Continuous wavelet transform and as well as discrete wavelet transform. An example of ECG waveform is shown in figure 1 .

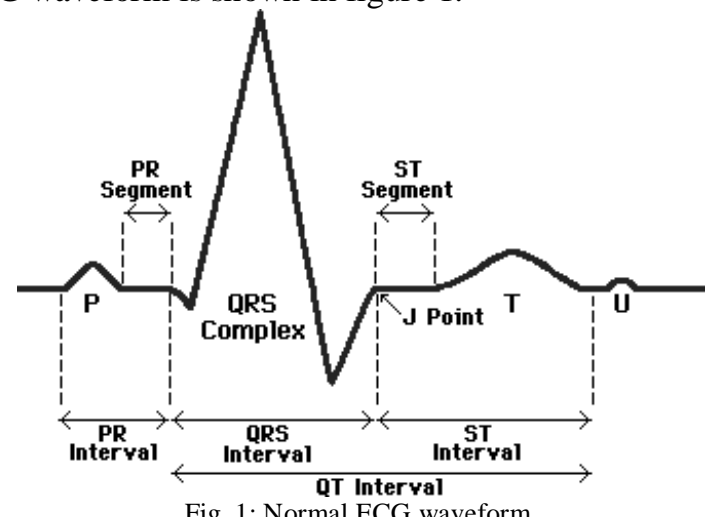

Fig. 1: Normal ECG waveform.

\section{LITERATURE REVIEW}

P.M. Dudhat, N.P. Joshi , D.S. Pipalia [1], proposed a portable ECG system ,in this paper feature extraction is done by performing several operations on the data like filtering, differentiation, squaring and time averaging of the signal. For processing the information and to perform operations they have used ARM cortex A8 processor and a 
DSP processor which is a OMAP 3530 application based processor which is used for signal conditioning on ECG data and adopted QRS detection algorithm based on matched filter to remove noises in the ECG data. After processing, The data has been sent through wireless communication network by means of a USB dongle, they've sent the ECG data to a remote health care centre and theses signals were analysed by physicians so that doctor can login into portable ECG device remotely and read and analyse it to reach conclusion that whether the patient is suffering from arrhythmia or not.

A.K.M Fazul Haque [2], devloped FFT and wavelet method for the extraction of small variations of the signal and shown that proposed wavelet method is superior to the conventional FFT in finding the small abnormalities in ECG signal. In this paper both standard and noise corrupted ECG signals have been generated using MATLAB and are analysed by the wavelet method using MATLAB wavelet tool and employed a 1-D discrete wavelet transform for decomposition process and feature extraction is done using FFT and wavelet method to show that proposed method is superior in finding small abnormalities in ECG signal.

Designing of feed forward neural network with the effect of artificial neural network parameter for feature extraction of ECG signal by employing wavelet decomposition was proposed by Dr. A.k.wadhwari and priyanka agarwal [3] .They employed Daubechies wavelet to decompose the ECG signal in to its higher and lower frequency components and then statistical feature of all components are given as input to neural network. A multilayer feed forward neural network employing a back propagation algorithm was used for learning and training the artificial neural network. They've designed and trained artificial neural network using MATLAB software which is provided with tools for analysis and reconstruction of signals and images using wavelets within the MATLAB domain .while selecting wavelet they considered one of the key criteria of selection of wavelet is its ability to fully reconstruct the signal from wavelet decomposition and hence chosen db6 because of its highest correlation coefficient with ECG waveform and its energy spectrum is also concentrated around low frequencies, while denoising the ECG, frequency domain filtering is performed by using discrete wavelet transform. DWT may also be considered as decomposition by wavelet filter banks and $\mathrm{R}$ peak are identified by writing a suitable MATLAB code. Back propogation algorithm is selected as learning algorithm for training and learning of artificial neural network.

Priyanka Mundhe, Harsha Bodhey, Prof. Anuja Diggikar [4], proposed an algorithm for QRS complex detection. In their proposed algorithm the QRS complex is detected by background wondering and noise reduction. The background wondering and noise reduction is done with the help of mathematical morphology. Morphological operators have been widely used in the signal and image processing fields because of their robust and adaptive performance in extracting the shape information in addition to their simple and quick set computation. In this technique the combined opening and closing operators for baseline correction of ECG signals are used and good filtering performance is obtained. Mathematical morphology, based on sets operations, provides an approach to the development of non-linear signal processing methods, in which the shape information of a signal is incorporated There are two most basic morphological set transformation operators: dilation and erosion, which all other mathematical morphology operations are based on.in their paper it is concluded that FPGA based morphology algorithm for both resting and wearable exercise ECG QRS detection in BSNs is presented and also shown that the method can also work in case of the bandwidth overlaps between QRS complex and other components. The proposed algorithm has been implemented in FPGA and the proposed detector compares very favourably with published results of other QRS detection algorithms. Dr. R.S.D. Wahidabanu and P. Sasikala devloped a robust $\mathrm{R}$ peak QRS detection using wavelet transform [5]. Discrete wavelet Transform (DWT) has been used to extract relevant information from the ECG signal in order to perform classification. In their application Daubechies4 wavelet has been selected for extracting the ECG features ECG signals required for analysis are collected from Physionet MIT-BIH arrhythmia database where annotated ECG signals are described by a text header file (.hea), a binary file (.dat) and a binary annotation file (.atr). The database contains 48 records, each containing two-channel ECG signals for $30 \mathrm{~min}$ duration selected from 24-hr recordings of 47 different individuals. Header file consists of detailed information such as number of samples, sampling frequency, format of ECG signal, type of ECG leads and number of ECG leads, patient's history and the detailed clinical information. In binary data signal file, the signal is stored in 212 format which means each sample requires number of leads times 12 bits to be stored and the binary annotation file consists of beat annotations. Signals were sampled using a 12-bit analog-to-digital converter board (National Instruments, PCI-6071E). Matlab and its wavelet toolbox were used for ECG Signal processing and Analysis. Analysis was performed on the PQRST waveform. In their work ECG signal is decomposed into several sub bands by applying wavelet transform and then each coefficient is modified by applying a threshold function and finally reconstructs the denoised signal. The detection of the QRS complex is based on modulus maxima of the Wavelet Transform. This is because modulus maxima and zero crossings of the Wavelet Transform correspond to the sharp edges in the signal. The QRS complex is also used for beat detection and the determination of heart rate through $R-R$ interval estimation. This information can also serve as an input to a system that allows automatic cardiac diagnosis.

Awadhesh Pachauri and Manabendra Bhuyan proposed an algorithm [6] for R-wave detection using wavelet 
technique has been developed. In this detection process, the importance of using a particular type of linear transform i.e. wavelet transform has been highlighted in which the noise is filtered at each level of decomposition eliminating the requirement of any preprocessing. This ensures the robustness of the method which has been further confirmed using different records of the database embedded with noise. Moreover, the dominancy of details of $\mathrm{R}$ wave in $\mathrm{d} 4$ level was further confirmed by Fourier transform. The time domain relationship between the $\mathrm{d} 4$ and the original signal was also confirmed by cross correlation analysis. The technique can be employed in the extraction of other ECG features and classification of various heart related diseases. The results with db6 have been found to be more stable by varying threshold than sym11 which picks up false peaks. The effect of zero padding has also been eliminated during energy analysis making the algorithm simpler and less time consuming.

Alan and Nikola [7] proposed chaos theory that can be successfully applied to ECG feature extraction. They also discussed numerous chaos methods, including phase space and attractors, correlation dimension, spatial filling index, central tendency measure and approximate entropy. They created a new feature extraction environment called ECG chaos extractor to apply the above mentioned chaos methods. A new semi-automatic program for ECG feature extraction has been implemented and is presented in this article. Graphical interface is used to specify ECG files employed in the extraction procedure as well as for method selection and results saving. The program extracts features from ECG files.

\section{III.CONCLUSION}

This paper provides an overview of the various techniques and algorithms for feature extraction of ECG signal proposed earlier in literature. The analysis of ECG signal depends upon the accurate detection of various features of ECG signal. In this review paper some of the important algorithm of ECG feature extraction presented in the past has been discussed. From the discussion it is clear that wavelet transform is one of the important tools for extracting out QRS complex and other features from the ECG signal. Feature extraction of ECG signal has the advantage of a direct link between arrhythmias and symptoms, which makes it possible to diagnose relevant arrhythmias.

\section{REFERENCES}

[1] P M Dudhat, N P Joshi , D S Pipalia Yiu Chan, Enderle J. D.," Designing scheme for portable ECG system with Real time application", IOSR Journal of Electronics and communication Engineering, Jan-Feb.

[2] A.K.M Fazlul Haque, "Improved detection of ECG features using wavelet for emergency medical application". IJAITI, march-April 2012.

[3] Dr. A.k Wadhwani , priyanka agarwal, " Neural network architecture design for feature extraction of ECG by wavelet ", international journal of computational engineering research, march-April 2012.

[4] Priyanka mundhe, Harsha bodhey "Implementation of ECG QRS complex detector for body sensor networks by using nano FPGA". International journal of computer technology and Electronics engineering. March-April 2013. 